\title{
Intraday Volatility of Stock Index Futures Analyzing via High Frequency Data
}

\author{
Li Yuan ${ }^{1,2, ~ a *, ~ Y o n g p i n g ~ G u i ~ 1, b ~}$ \\ ${ }^{1}$ Department of Finance, School of Economics, Shanghai University, Shanghai City, China) \\ ${ }^{2}$ School of Mechatronic Engineering and Automation, Shanghai University, Shanghai City, China
}

Keywords: Stock Index Futures, Intraday Volatility.

Abstract. This paper presents a method for measuring the intraday volatility of the stock index futures, which uses a multi-resolution histogram to describe the price volatility in different time scales. The high frequency data are deployed to show the price volatility during a day. The long term relationship between the trade volume and the price volatility is also given. Experiments show that this method can be used to provide quantitative reference for analysis of transaction behaviors in the market.

\section{Introduction}

Stock index futures is one of the commonly used financial risk management tools in the international market. Many literatures show that stock index futures as an effective tool to avoid the volatility in the market [1]. However, the large number of quantitative trading clients produces a huge fluctuation in the market. From the world, the similar cases have occurred many times. For example, in 1987 the U.S. stock market crash, in subsequent studies, stock index trading is considered to be one of the main reasons for the rapid decline in stock prices [2].

In the past 5 years, the stock index futures trading volume continued to increase in China. The peak of the daily trading volume has reached more than two million in July 2015. More than $90 \%$ of the transactions are completed in the same day, which indicates that it is a speculative market. More and more people in the market believe that the quantitative trading system help them to give a rapid response to the market price changes. This fact may ultimately to form many extreme price movements. In September 2015 after the new transaction limit rules are implemented in China market, the daily trading volume dropped to about 10,000.

The current theoretical research is mainly focused on the relationship between stock price index and the price of the two markets. There is no clear conclusion about the impact of the trading volume increasing to the market itself. In this paper, an improved Dynamic Histogram Multiresolution $(\mathrm{MRDH})$ is presented to describe the intraday volatility of stock index futures price within different time scales, which can characterize the multi-scale features of price volatility in certain period, and to quantify the impact of trading volume on the volatility of stock index futures market.

\section{Related Works}

A lot of studies have considered that the high frequency trading will make a negative impact on price formation mechanism and lead to market risk. For example, Alain et al. [3] find that a high correlation of trading algorithm is easy to cause the price fluctuations. Kirilenko et al. [4] show that the high-frequency trading will change the average market price without any new information. Zhang [5] analyzes the Thomson and Reuters CRSP database in the stock and find that there is a positive correlation between the high frequency trading and stock price volatility when the company's basic surface changes and other external influence are removed from the data.

Other researchers have found that the high frequency trading has little or no effect on price discovery mechanism. For example, Brogaard [6] believes that the high-frequency trading has brought only substantial increase in price discovery mechanism. However, most high frequency trading make the price has the characteristics of mean reversion, which means it can play a role in stabilizing prices. 
In fact, it is difficult to discriminate which order is sent by a program terminal or a traditional trader. The traditional method for volatility measurement, such as The Average True Range (ATR) and the OHLC method are suitable for the long term price volatility, which are not able to clearly describe the high frequency trading. The researchers begin to use the real data from stock index futures market to study the intraday volatility effects of China market [7], the volatility measures [8], and the relationship between stock index futures and stock index futures [9], especially based on the analysis of the high frequency data. Those researches are helpful to gain insight into the behavior of asset price and reveal the essential characteristics of stock index futures market.

\section{Volatility Description}

In order to describe the relationship between price fluctuation of stock index futures and the status of market, we give a multi-resolution description for describe the of price volatility. The corresponding computational methods are also given.

First, we calculate the probability measures of the high frequency price data. This measure $h(t)$ can be expressed by a histogram. The price of the $t$ is divided into $n$ regions (bins) from low to high. The value of $n$ is changed with the price distribution of $t$ which means the histogram is dynamic changed with the current market status. The width of each bin is $\delta=\kappa p_{t}$, where the $p_{t}$ is a minimum price jump variable (size tick), $\kappa$ is an integer coefficient, generally take $1 . h(t)$ contains a set of statistical sequences $\mathbf{v}=\left\{v_{i}\right\}, 0<i<n$, where $v_{i}$ means the frequency of the $i$ group of price. It defined

$$
v_{i}=\sum_{j=1, p_{j} \in P_{i}}^{N} \frac{1}{N}
$$

Where $P$ is a collection of all the prices within the period $t$, and $N$ is the total number of elements within the $P$. This design makes the width of the histogram can be adjusted automatically with the increase of the price, which can maintain the consistency in various price ranges. On the whole, the histogram $h(t)$ describes the dynamic distribution of the price of the time $t$.

The width of $h(t)$ is $W=\delta \times n$. It is a 1-order expression of the price volatility within time $t$. The change rate of $W$ indicates the magnitude of the price fluctuation in a time scale. A sharp increase in volatility indicates that the process of buying or selling in the market. The decrease in the volatility means the market is dull. The amplitude distribution of $h(t)$ is the expression of the stability of the market, which has nothing to do with the current price. The position of the peak amplitude and the direction of movement indicate the current price trend.

Based on the description, it is convenient to construct a set of multi-resolution histogram by setting different range $H(T)=\left\{h_{i}\left(t_{i}\right)\right\}$ to describe the degree of price volatility in different time scales. Among them, $t_{i}$ is a series of time intervals for characterizing of the volatility of different time window. In fact, the different rates can be calculated by using the same form. Fig. 1 shows multiple time scale descriptors. Fig. 1 (b) shows the price of a day. Fig. 1 (a), (c) - (f) are volatility descriptors for 1 minutes, 3 minutes, 10 minutes and 30 minutes, respectively.

In order to give an intuitive evaluation of the daily price volatility of stock index futures, we use of $h(x)$ to define two volatility measures $I_{o}$ and $I_{i}$. The former expresses the range of the macro volatility of the day, and the latter describe of microstructure fluctuations. $I_{d}$ is expressed by $I_{d}=f_{b} / f_{o} W$,where $f_{o}$ express opening price, $f_{b}$ indicates a predefined benchmark price. The coefficient $f_{b} / f_{o}$ is used to balance the price of different days. $W$ is the width of $h(x)$. When $f_{o}=f_{b}$, $I_{d}$ is same as ATR. $I_{i}$ is defined as the weighted sum of the price volatility from a set of different time

scales.

$$
I_{i}=\sum_{k=1}^{M}\left(\alpha_{k} d_{k}(\sigma)\right)
$$

As shown in fig. 2 (a), the value of a function $d_{k}(\sigma)$ is defined by a fixed time scale, where $\sigma$ is the 
variance of the selection of a Gauss filter. The weight $\alpha_{k}$ is the ratio of the total price of the current price set that can be obtained by formula (1).

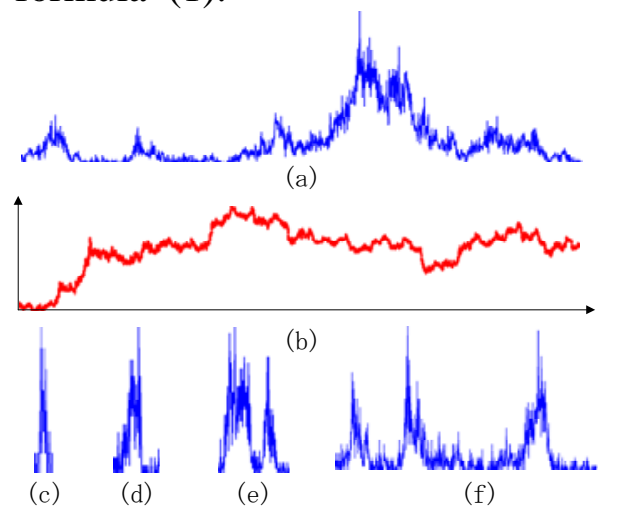

Figure 1. Multi-scale descriptor for price volatility

Since price volatility can be described on a number of scales, we describe the effective price volatility as the distance between the statistical and the price peak. Fig. 2 (b) shows the price of IF1507 contract in July 3, 2015. Fig. 2 (c) gives the value of $I_{i}$ with $\sigma=3, \sigma=3$, and $\sigma=3$ respectively.

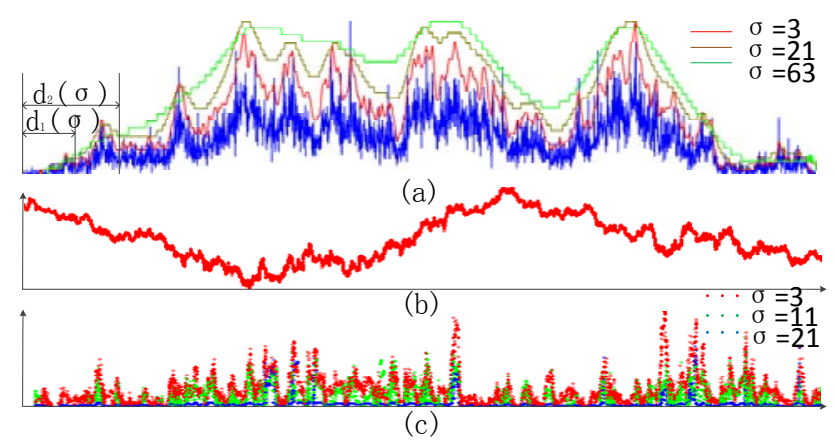

Figure 2. Price volatility measurement

In order to calculate the index in real time, we use histogram dynamic update method to avoid the repeatedly statistics and generate a histogram. Therefore, the computation time is constant when generate the histogram for different scales value. To compute $d_{k}(\sigma)$, we get the distribution troughs by computing the derivation of one dimensional Gaussian derivatives to generate multiscale volatility curve fitting and get different scales of fluctuation range, which make the computation fast and stable.

\section{Experiment and discussion}

We analyze the correlation between the trading volume and price vilotility in China stock index futures market with $I_{o}$ and $I_{d}$. Experimental data is selected from 2012 to 2015 of the stock index futures contract with high frequency sampling (2-3 per second).

In the experiment, the daily volatility is express by $I_{i}$ and $I_{d}$. In fig. 3 , the horizontal axis is the trading day, the vertical axis is the volatility index of $I_{i}$ and $I_{d}$. The setting of the $\sigma$ value is the same as that in Fig. 2 (a). As shown in fig. 3, on the small time scales, $I_{1}(\sigma=3)$ and $I_{2}(\sigma=21)$ changed very intense. $I_{3}(\sigma=63)$ is close to the macro volatility $I_{d}$.

Fig. 4 shows the trend of trading volume from the stock index futures IF contract from 2012 to 2015. In order to maintain the consistency of the analysis, the experiment get rid of the last 15 minutes of trading data. As shown from fig. 4, the daily trading volume changing in 2012 to January 2014 is relatively stable. However, from January 2014 large fluctuations often appears. The corresponding $I_{1}$ and $I_{2}$ are also appear large fluctuation. From the analysis of fig. 3 and fig. 4, we can see that there is no obvious change on $I_{d}$ when the trading volume changes. However, there are high correlation between $I_{i}$ and the trading volume. After September 2015, the correlation is weakened, which means the trading volume on price volatility has been weakened after the execution of the new rules. 


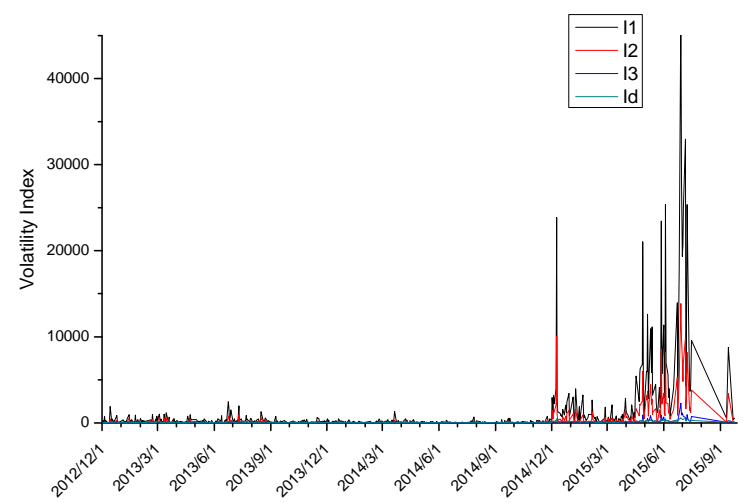

Figure 3. Multi-scale price volatility

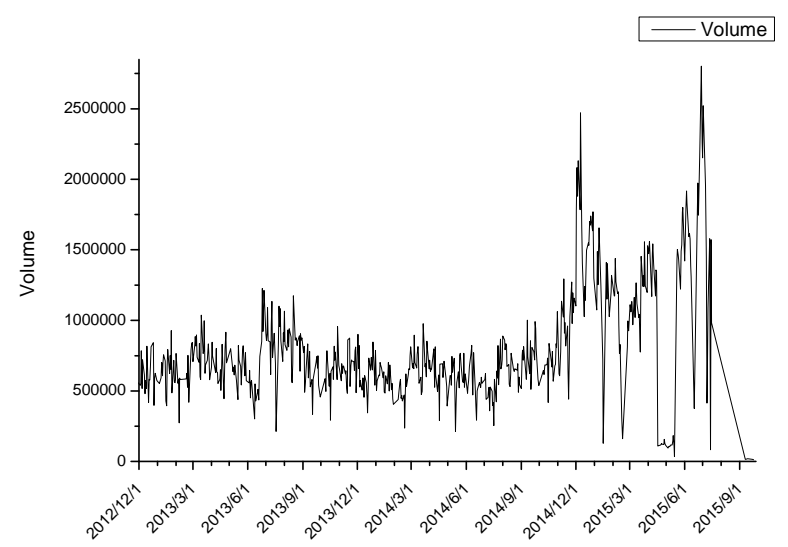

Figure 4. Daily trading volume

\section{Summary}

In this paper, the MRDH is used to describe intraday volatility of the trading day. Compared with ATR, it provides more accurate measurement. Furthermore, it can reflect the micro morphology of the market price trend in high frequency trading, especially to reflect how the market reacts when after special external news. Based on the MRDH, this paper analyzes the characteristics of trading volume and price volatility in the days using the high frequency data from the stock index futures market of China.

The model given in this paper is a description of the volatility of the market price, which provides measuring tools for quantitative analysis of high frequency trading data. However it cannot describe the market trend in the future.

\section{References}

[1] S. Zhang, The Impact of CSI300Stock Index Futures on the Volatility of the Spot Market in China, Master Thesis, Jinan University, 2013, In Chinese.

[2] N. F. Brady, Report of the Presidential Task Force on Market Mechanisms, U.S. Government Printing Office, 1988.

[3] A. P. Chaboud, B. Chiquoine, et al. Rise of the machines: Algorithmic trading in the foreign exchange market. The Journal of Finance, 69(5): 2045--2084.

[4] J. Cvitanic, A A. Kirilenko, High Frequency Traders and Asset Prices, Social Science Research Network, 2010.

[5] F. Zhang, High-frequency trading, stock volatility, and price discovery, Available at SSRN 1691679. 2010.

[6] J. A. Brogaard, High frequency trading and its impact on market quality, Northwestern University Kellogg School of Management Working Paper (2010): 66.

[7] Y. Sun, M J. He, W. Zhou, Empirical Research on the Stock Index Market Based on the UHF-EGARCH Model, Journal of Management Science, 2011(06): 113-120, In Chinese.

[8] R. Long, C. Xie, et al. Measurement of CSI 300 stock index futures volatility under high-frequency environment - Methods based on realized volatility and its modification, Systems Engineering - Theory \& Practice, 2011(05): 813-822, In Chinese.

[9] X. Y. Zhang, D P. Ren, The Price-Volume Relation of the CSI 300 Stock Index Futures: Evidences from High Frequency Data, Journal of Hunan University (Social Sciences). 2013(02): 48-54, In Chinese. 